\title{
The molecular gas in Luminous Infrared Galaxies: a new emergent picture
}

\author{
Padelis P. Papadopoulos ${ }^{1}$, Zhi-Yu Zhang ${ }^{2}$, Axel Weiss ${ }^{1}$, Paul van der \\ Werf $^{3}$, Kate Isaak ${ }^{4}$, Yu Gao ${ }^{2}$, Manolis Xilouris ${ }^{5}$, \\ and Thomas R. Greve ${ }^{6}$ \\ ${ }^{1}$ Max Planck Institute for Radioastronomy, Auf dem Hügel 69, D-53121 Bonn, Germany, \\ email: padelis@mpifr-bonn.mpg.de \\ ${ }^{2}$ Purple Mountain Observatory, Chinese Academy of Sciences, Nanjing, Jiangsu 210008, China \\ ${ }^{3}$ Leiden Observatory, Leiden University, NL-2300 RA Leiden, The Netherlands \\ ${ }^{4}$ Research \& Scientific Support, European Space Agency, ESTEC, NL-2201, The Netherlands \\ ${ }^{5}$ Institute for Astronomy, Astrophysics, Space Applications \& Remote Sensing, National \\ Observatory of Athens, P. Penteli, 15236 Athens, Greece \\ ${ }^{6}$ Department of Physics and Astronomy, University College London, London WC1E 6BT, UK
}

\begin{abstract}
Results from a large, multi-J CO, ${ }^{13} \mathrm{CO}$, and $\mathrm{HCN}$ line survey of Luminous Infrared Galaxies (LIRGs: $\mathrm{L}_{I R} \geqslant 10^{10} \mathrm{~L}_{\odot}$ ) in the local Universe ( $\mathrm{z} \leqslant 0.1$ ), complemented by CO $\mathrm{J}=4-3$ up to $\mathrm{J}=13-12$ observations from the Herschel Space Observatory (HSO), paints a new picture for the average conditions of the molecular gas of the most luminous of these galaxies with turbulence and/or large cosmic ray (CR) energy densities $U_{\mathrm{CR}}$ rather than far-UV/optical photons from star-forming sites as the dominant heating sources. Especially in ULIRGs $\left(\mathrm{L}_{\mathrm{IR}}>10^{12} \mathrm{~L}_{\odot}\right)$ the Photon Dominated Regions (PDRs) can encompass at most a few \% of their molecular gas mass while the large $\mathrm{U}_{\mathrm{CR}} \sim 10^{3} \mathrm{U}_{\mathrm{CR}, \text { Galaxy }}$, and the strong turbulence in these merger/starbursts, can volumetrically heat much of their molecular gas to $\mathrm{T}_{\text {kin }} \sim(100-200) \mathrm{K}$, unhindered by the high dust extinctions. Moreover the strong supersonic turbulence in ULIRGs relocates much of their molecular gas at much higher average densities $\left(\geqslant 10^{4} \mathrm{~cm}^{-3}\right)$ than in isolated spirals $\left(\sim 10^{2}-\right.$ $\left.10^{3} \mathrm{~cm}^{-3}\right)$. This renders low-J CO lines incapable of constraining the properties of the bulk of the molecular gas in ULIRGs, with substantial and systematic underestimates of its mass possible when only such lines are used. Finally a comparative study of multi-J HCN lines and CO SLEDs from $\mathrm{J}=1-0$ up to $\mathrm{J}=13-12$ of NGC 6240 and Arp 193 offers a clear example of two merger/starbursts whose similar low-J CO SLEDs, and $\mathrm{L}_{\mathrm{IR}} / \mathrm{L}_{\mathrm{CO}, 1-0}$ and $\mathrm{L}_{\mathrm{HCN}, 1-0} / \mathrm{L}_{\mathrm{CO}, 1-0}$ ratios (proxies of the so-called SF efficiency and dense gas mass fraction), yield no indications about their strongly diverging CO SLEDs beyond $\mathrm{J}=4-3$, and ultimately the different physical conditions in their molecular ISM. The much larger sensitivity of ALMA and its excellent site in the Atacama desert now allows the observations necessary to assess the dominant energy sources of the molecular gas and its mass in LIRGs without depending on the low-J CO lines.
\end{abstract}

Keywords. techniques: spectroscopic — galaxies: ISM — galaxies: starburst — ISM: molecules — cosmic rays - ISM: radio lines

\section{Introduction}

The CO rotational lines are the most widely used probes of the average conditions and mass of the molecular gas in galaxies, with a substantial body of data assembled over the last two decades (e.g. Braine \& Combes 1992; Aalto et al. 1995; Solomon et al. 1997, Downes \& Solomon 1998, Mauersberger et al. 1999 Yao et al. 2003, Mao et al. 2011). Most of these are for $\mathrm{J}=1-0,2-1$, with only few datasets having $\mathrm{J}=3-2$ or higher- $\mathrm{J}$ lines. 
The lowest $\mathrm{J}=1-0$ line serves as a global molecular gas mass tracer via the so-called $\mathrm{X}_{\mathrm{CO}}=\mathrm{M}\left(\mathrm{H}_{2}\right) / \mathrm{L}_{\mathrm{co}, 1-0}^{\prime}$ factor (Dickman et al. 1986; Solomon \& Barrett 1991). The low excitation requirements of this transition $\left(\mathrm{E}_{1} / \mathrm{k}_{\mathrm{B}} \sim 5.5 \mathrm{~K}\right.$ and $\left.\mathrm{n}_{\text {crit }} \sim 400 \mathrm{~cm}^{-3}\right)$ allow even the coldest and lowest density gas in ordinary Giant Molecular Clouds (GMCs) to have a substantial, and hopefully calibratable, line luminosity contribution.

The next two decades were then spent formulating the dependance of $\mathrm{X}_{\mathrm{CO}}$ on the average molecular gas conditions, and calibrating its values for various ISM environments (Maloney \& Black 1988, Young \& Scoville 1991, Wolfire et al. 1993, Bryant \& Scoville 1996, Downes \& Solomon 1998). Aside from a strong dependance of $\mathrm{X}_{\mathrm{CO}}$ on metallicity that could leave large reservoirs of molecular gas in the outer parts of spirals or metalpoor dwarfs untraceable by CO (Papadopoulos et al. 2002, Wolfire et al. 2010), any deviations of $\mathrm{X}_{\mathrm{CO}}$ from its Galactic value of $\sim 5 \mathrm{M}_{\odot}\left(\mathrm{K} \mathrm{km} \mathrm{s}^{-1} \mathrm{pc}^{2}\right)^{-1}$ were determined using radiative transfer models of $\mathrm{CO}$ lines to constrain the average gas density $\mathrm{n}\left(\mathrm{H}_{2}\right), \mathrm{CO}$ $\mathrm{J}=1-0$ brightness temperature $\mathrm{T}_{\mathrm{b}, 1-0}$, and dynamical state upon which $\mathrm{X}_{\mathrm{co}}$ depends as

$$
\mathrm{X}_{\mathrm{CO}}=\frac{\mathrm{M}\left(\mathrm{H}_{2}\right)}{\mathrm{L}_{\mathrm{co}, 1-0}^{\prime}}=\frac{3.25}{\sqrt{\alpha}} \frac{\sqrt{\mathrm{n}\left(\mathrm{H}_{2}\right)}}{\mathrm{T}_{\mathrm{b}, 1-0}} \mathrm{~K}_{\mathrm{vir}}^{-1}\left(\frac{\mathrm{M}_{\odot}}{\mathrm{Kkm} \mathrm{s}^{-1} \mathrm{pc}^{2}}\right),
$$

(Papadopoulos et al. 2012a) where $\alpha=0.55-2.4$ depending on the cloud density profile, and $\mathrm{L}_{\mathrm{co}, 1-0}^{\prime}=\int_{\Delta \mathrm{V}} \int_{\mathrm{A}_{\mathrm{s}}} \mathrm{T}_{\mathrm{b}, 1-0} \mathrm{dadV}$ is the velocity/area-integrated CO $\mathrm{J}=1-0$ brightness temperature at the reference frame of the source. The parameter $\mathrm{K}_{\mathrm{vir}}$ is given by

$$
\mathrm{K}_{\mathrm{vir}}=\frac{(\mathrm{dV} / \mathrm{dR})}{(\mathrm{dV} / \mathrm{dR})_{\text {virial }}} \sim 1.54 \frac{\left[\mathrm{CO} / \mathrm{H}_{2}\right]}{\sqrt{\alpha} \Lambda_{\mathrm{co}}}\left(\frac{\mathrm{n}\left(\mathrm{H}_{2}\right)}{10^{3} \mathrm{~cm}^{-3}}\right)^{-1 / 2}
$$

where $\left[\mathrm{CO} / \mathrm{H}_{2}\right] \sim 10^{-4}$ is the $\mathrm{CO}$ abundance and $\Lambda_{\mathrm{co}}=\left[\mathrm{CO} / \mathrm{H}_{2}\right] /(\mathrm{dV} / \mathrm{dR})$ is one of the three parameters (the other two being $\mathrm{n}\left(\mathrm{H}_{2}\right)$ and $\mathrm{T}_{\text {kin }}$ ) of one-phase Large Velocity Gradient (LVG) radiative transfer models (with $\mathrm{dV} / \mathrm{dR}$ the gas velocity gradient). The values of $K_{v i r}$ determine the average gas dynamical state, with $K_{v i r} \sim 1-2$ typical of selfgravitating (or nearly so) states, and $\mathrm{K}_{\mathrm{vir}} \gg 1$ indicating unbound gas (Papadopoulos et al. 2012a). In principle, LVG models of the lowest three CO lines can provide constraints on $\mathrm{X}_{\mathrm{CO}}$ to within factors of $\sim 2$ via Equation 1.1 (or via similar expressions in the literature, e.g. Bryant \& Scoville 1996) since the excitation characteristics of $\mathrm{J}=3-2$ $\left(\mathrm{E}_{3} / \mathrm{k}_{\mathrm{B}}=33 \mathrm{~K}, \mathrm{n}_{\mathrm{crit}} \sim 10^{4} \mathrm{~cm}^{-3}\right)$ bracket the conditions expected for much of the gas in GMCs $\left(\mathrm{T}_{\mathrm{kin}} \sim(10-30) \mathrm{K}, \mathrm{n} \sim\left(500-10^{3}\right) \mathrm{cm}^{-3}\right)$. For merger/starbursts such studies yielded a low $\mathrm{X}_{\mathrm{co}} \sim 1 \mathrm{M}_{\odot}\left(\mathrm{K} \mathrm{km} \mathrm{s}^{-1} \mathrm{pc}^{2}\right)^{-1}$ (Solomon et al. 1997, Downes \& Solomon 1998, Yao et al. 2003), which was then widely used in the literature for similar galaxies at low or high redshifts.

The $\mathrm{X}_{\mathrm{co}}$ dependence on the physical conditions of GMCs brings forth the question of what determines them (and thus $\mathrm{X}_{\mathrm{co}}$ ) in LIRGs. When it comes to the all-important thermal state, far-UV/optical photons from star-forming (SF) sites have long been considered as the main heating source of the molecular gas and dust in the Galaxy, with PDRs containing $\sim 90 \%$ of its molecular gas in Photon Dominated Regions (PDRs) where photons determine chemistry and thermal balance (Hollenbach \& Tielens 1999). The molecular ISM and its corresponding line and dust continuum in LIRGs is then considered fully reducible to PDR ensembles (Wolfire et al. 1990).

\section{The line survey of LIRGs: reaching out to the warm and dense gas}

We undertook a large molecular line survey of LIRGs, drawn from the IRAS Bright Galaxy Survey (BGS) (Soifer et al. 1987; Sanders et al. 2003) using the James Clerk 

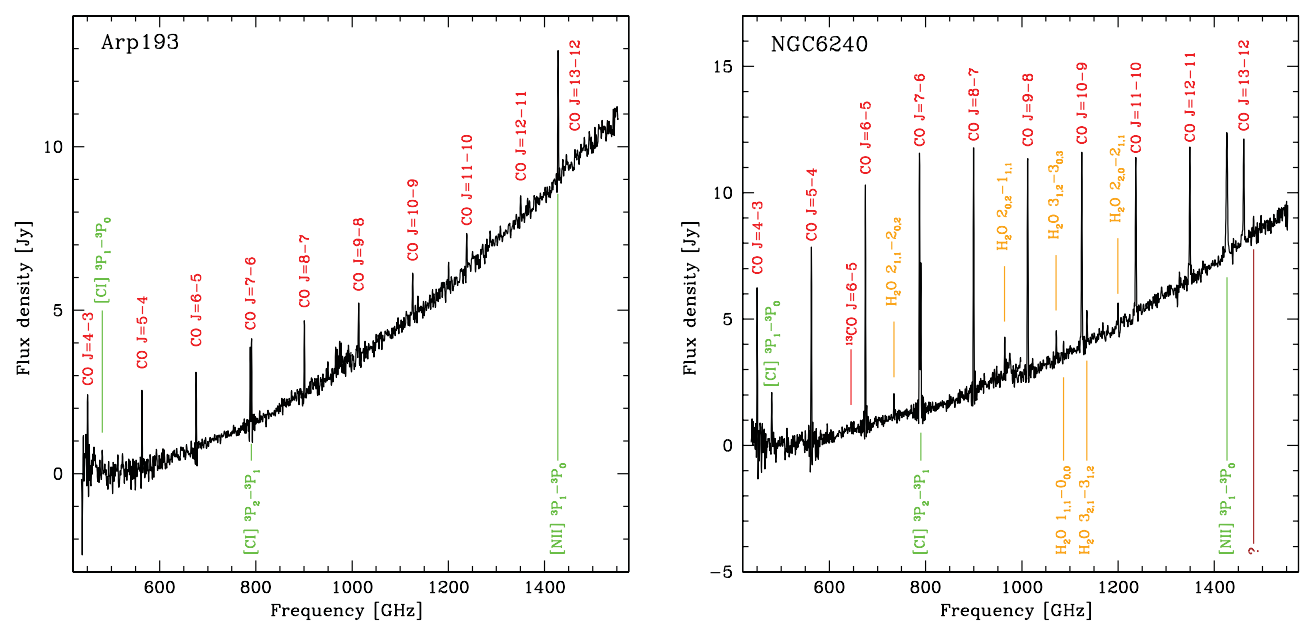

Figure 1. The SPIRE/FTS spectra of Arp 193, NGC 6240. The lines are: CO J=4-3 up to $\mathrm{J}=13-12$ (red), the two fine structure lines of [C I $]^{3} P_{1} \rightarrow{ }^{3} P_{0}$ and ${ }^{3} P_{2} \rightarrow{ }^{3} P_{1}$ and [N II] (green).

Maxwell Telescope (JCMT) on Mauna Kea in Hawaii, and the IRAM 30-m telescope on Pico Veleta in Spain. Our line database, when augmented by data existing in the literature, yielded the largest multi-J dataset of total $\mathrm{CO},{ }^{13} \mathrm{CO}$ and $\mathrm{HCN}$ line luminosities for galaxies in the local $(\mathrm{z} \leqslant 0.1)$ Universe (Papadopoulos et al. 2012b). The lines are CO $\mathrm{J}=1-0,2-1,3-2,{ }^{13} \mathrm{CO} \mathrm{J}=1-0$ and/or $\mathrm{J}=2-1$, as well as $\mathrm{HCN} \mathrm{J}=1-0,3-2$, and $4-3$, while for a smaller subsample $\mathrm{CO} J=4-3$ and $\mathrm{J}=6-5$ are also available. This groundbased effort has been complemented by SPIRE/FTS observations of a large number of ULIRGs in our sample, yielding complete CO SLEDs from $\mathrm{J}=1-0$ up to $\mathrm{J}=13-12$, and an unparalleled view of the densest and warmest gas in these merger/starbursts (see Figure 1).

Even a casual inspection of the high-J CO SLEDs of the two merger/starbursts Arp 193 and NGC 6240 (Figure 1) reveals two systems that, despite their similar low-J CO SLEDs and $\mathrm{L}_{\mathrm{IR}} / \mathrm{L}_{\mathrm{CO}, 1-0}$ and $\mathrm{L}_{\mathrm{HCN}, 1-0} / \mathrm{L}_{\mathrm{CO}, 1-0}$ ratios (proxies of the so-called SF efficiency and dense gas mass fraction), they have strongly divergent CO SLEDs above $\mathrm{J}=4-3$, indicating different properties and/or mass for their warm and dense gas phase. This high-J CO SLED divergence appears even more clearly in Figure 2 where they are shown normalized by the corresponding IR luminosities. It is worth noting that the $\mathrm{CO} /{ }^{13} \mathrm{CO} \mathrm{J}=2-1$ line ratios for these two galaxies are also similar (Papadopoulos et al. 2012b).

Thus the entire low-J CO, ${ }^{13}$ CO line diagnostic that is typically used to constrain the average molecular gas properties (and the corresponding $\mathrm{X}_{\mathrm{co}}$ factor) in LIRGs may be inadequate for the merger/starburst systems among them. This is not unexpected since, unlike in isolated SF disk galaxies, the much stronger supersonic turbulence in the molecular gas of ULIRGs (e.g. Downes \& Solomon 1998) will relocate most of the resident molecular gas mass to average densities of $n \geqslant 10^{4} \mathrm{~cm}^{-3}$, and thus beyond the reach of the low-J CO, ${ }^{13} \mathrm{CO}$ lines. The latter then can no longer yield relevant corrections for the $\mathrm{X}_{\mathrm{co}}$ factor in these systems (Papadopoulos et al. 2012a).

For Arp 193 and NGC 6240, the only ground-based observations able to break the aforementioned degeneracies were multi-J HCN line observations (Papadopoulos 2007). These found a very low $\mathrm{HCN}(4-3) /(1-0)$ ratio in Arp 193, compatible with a total lack of gas at $n \geqslant 10^{4} \mathrm{~cm}^{-3}$, quite unlike the state of the gas in NGC 6240, and what is generally expected in such extreme merger/starbursts (Gao \& Solomon 2004). These diferences 


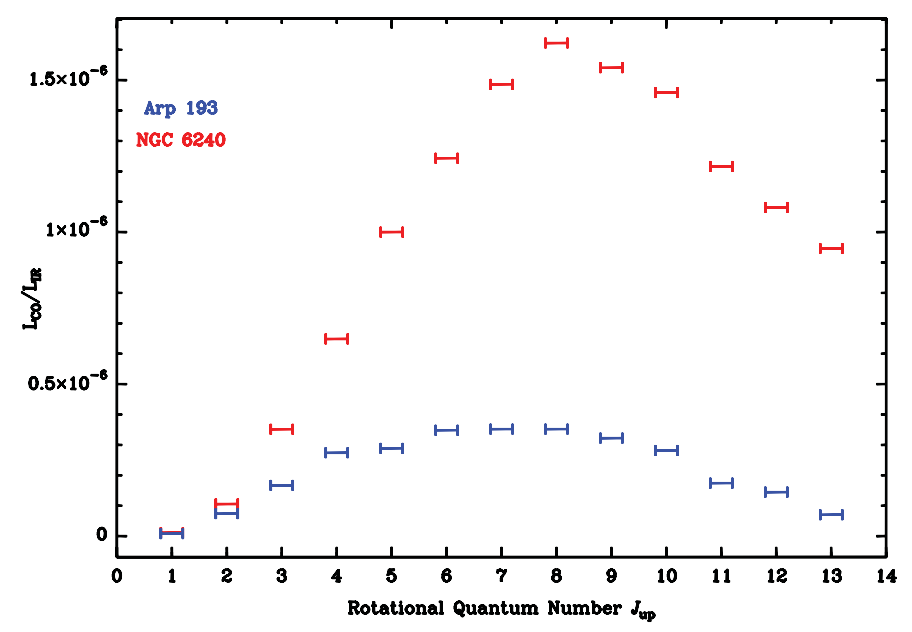

Figure 2. The CO SLEDs of Arp 193 and NGC 6240 normalized by their $\mathrm{L}_{\mathrm{IR}}\left(\mathrm{L}_{\odot} / \mathrm{L}_{\odot}\right.$ units).

are seen clearly in Figure 3 that shows the $\left[n\left(H_{2}\right), T_{k}\right]$ space compatible with the HCN line ratios of these two systems. In the past multi-J measurements of such high-dipole moment molecules were impractical because of the limited sensitivities of the available $\mathrm{mm} /$ submm telescopes. This will no longer be the case with ALMA, and multi-J HCN, $\mathrm{HCO}^{+}$, CS observations of (U)LIRGs may be the only way of assessing the average conditions of their molecular gas (and its mass) without the aforementioned degeneracies of the low-J CO lines (Papadopoulos et al. 2012a), and without depending on fullysampled high-J CO SLEDs. These will remain difficult to obtain past $\mathrm{J}=6-5$, even with ALMA, because of the large atmospheric absorption at $\nu \geqslant 690 \mathrm{GHz}$.

\section{New power sources for the molecular gas in ULIRGs}

In the high-density and metal-rich molecular ISM of ULIRGs neither far-UV/optical photons nor SNR-induced shocks travel far, the latter dissipating strongly in high density gas. The strong confinement of warm PDRs and SNR-shocked regions is evident even in the Galaxy where most of the molecular gas is at much lower average densities. Such regions involve only $\sim(0.1-1) \%$ of the mass of a typical GMC (Papadopoulos et al. 2012b), and this is indeed why even the template SF clouds of Orion A, B are globally cold with $\mathrm{CO}(2-1) /(1-0)$ ratios of $\sim 0.6-0.7$, and only few isolated regions near $\mathrm{H}$ II regions reaching $\sim 0.9-1.3$ (Sakamoto et al. 1994). The corresponding global $\mathrm{CO}(3-2) /(1-0)$ for Orion would then be $\sim 0.30$ while for the SF "hot-spots" $\mathrm{r}_{32} \sim 0.9-1.3$. Thus it is indeed a surprise that entire galaxies can approach and even surpass these high-excitation regimes (see Figure 4). These excitation outliers are typically merger/starburst LIRGs, and analysis of their $\mathrm{CO}$ ratios (often supplemented by available $\mathrm{CO} \mathrm{J}=4-3,6-5$ lines) find large $(\geqslant 10 \%)$ fractions of dense $\left(\sim 10^{4}-10^{5} \mathrm{~cm}^{-3}\right)$ and warm $(\sim(100-200) \mathrm{K})$ molecular gas.

For NGC 6240 and Arp 193 where HCN multi-J and fully sampled CO SLEDs from $\mathrm{J}=1-0$ up to $\mathrm{J}=13-12$ are available (Figure 1) a superposition of states from the HCNconstrained LVG solutions (Figure 3 ) with $\mathrm{T}_{\text {kin }} \geqslant 70 \mathrm{~K}$ is adequate to reproduce the entire CO SLEDs up to the highest transition. Only for the lowest two CO lines are there additional contributions from unbound low-density gas that contains little mass. For NGC 6240 in particular, the HCN-bright phases that can reproduce its luminous high-J CO SLED with its large line-continuum contrast (Figs 1, 2) have $\mathrm{n}\left(\mathrm{H}_{2}\right) \sim($ few $) \times 10^{4} \mathrm{~cm}^{-3}$, and contain most of its molecular gas mass. Far-UV photons cannot drive such high tem- 


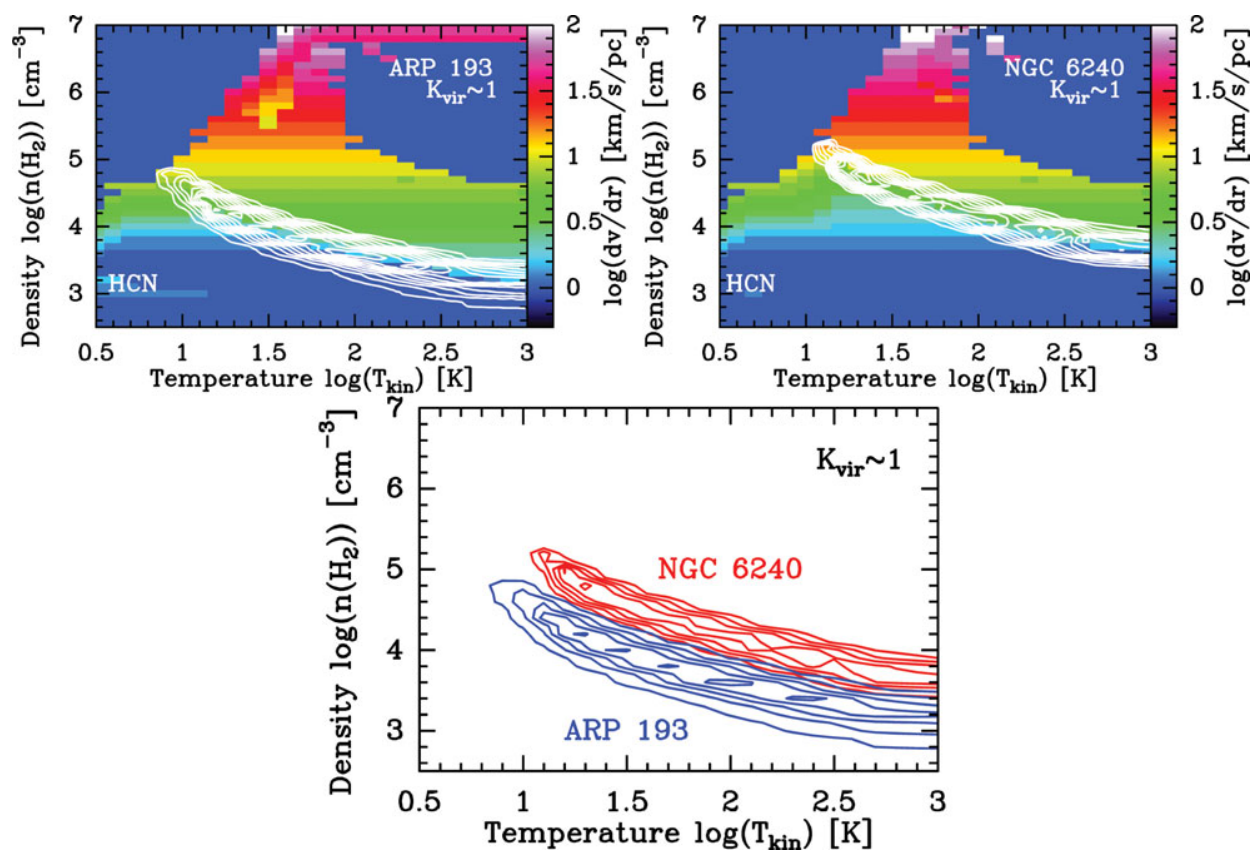

Figure 3. The two-dimensional probability density functions of the $\left[\mathrm{n}, \mathrm{T}_{\mathrm{kin}}\right] \mathrm{LVG}$ solutions in steps of 0.2 , as constrained by the HCN line ratios of NGC 6240 and Arp 193. Color: the corresponding $(\mathrm{dV} / \mathrm{dR})$ within the $\mathrm{K}_{\mathrm{vir}}=0.5-2$ range (Equation 1.2$)$.
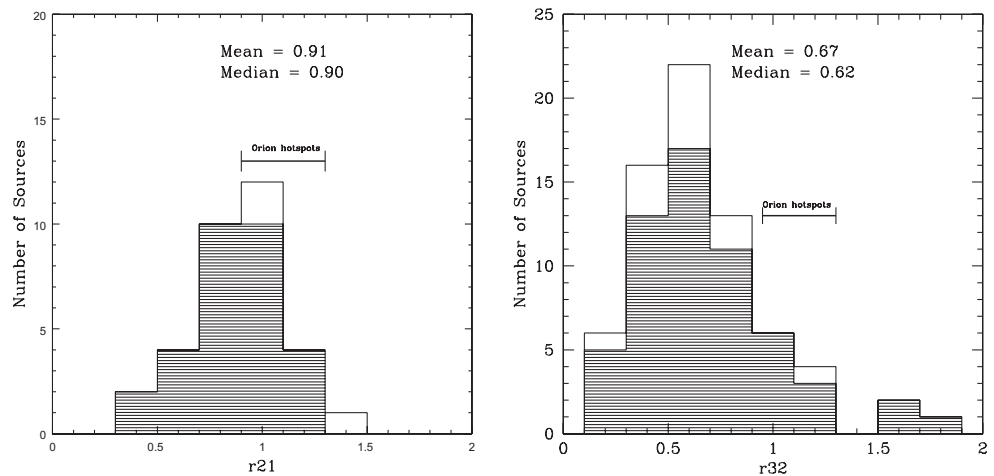

Figure 4. The distributions of the CO $(2-1) /(1-0)(3-2) /(1-0)$ brightness temperature ratios for the galaxies in the sample. The shaded area marks those for sources with CO emission sizes $\leqslant 15^{\prime \prime}$ (the JCMT beam). The horizontal bars indicate the ratios for the Orion SF "hot-spots".

peratures since, for photoelectric heating, $\Gamma_{\text {pe }} \ll \Lambda_{\mathrm{CO}}+\Lambda_{\mathrm{H}_{2}}+\Lambda_{\mathrm{O} \text { I }}+\Lambda_{\text {gas }- \text { dust }}$, because the line cooling terms (i.e. $\mathrm{CO}, \mathrm{H}_{2}$ and $\mathrm{OI}$ cooling) become large at high densities $\left(\Lambda_{\text {line }} \propto\left[\mathrm{n}\left(\mathrm{H}_{2}\right)\right]^{2}\right)$. On other hand, the strong supersonic turbulence and the high CR energy densities in the ISM environment of (U)LIRGs like NGC 6240 can easily maintain large amounts of warm and dense molecular gas mass (Papadopoulos et al. 2012a).

\section{A Galactic $\mathrm{X}_{\mathrm{CO}}$ in ULIRGs?}

Using Equation 1.1 with inputs from the HCN-constrained LVG solution space of NGC 6240 shown in Figure 3 (which also satisfies the multi-J CS and $\mathrm{HCO}^{+}$available for this LIRG) we obtain the $\mathrm{X}_{\mathrm{CO}}$ factor for the prevailing conditions of its molecular gas 


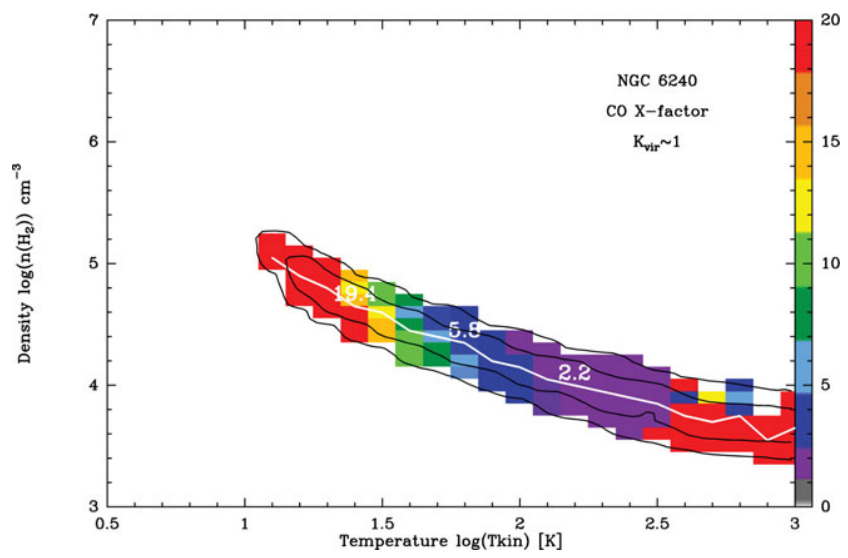

Figure 5. The $\mathrm{X}_{\mathrm{CO}}$ factor for NGC 6240 and the conditions shown in Figure 3.

(Figure 5). It is obvious that for the high-density molecular gas of this merger/starburst Galactic $X_{C O}$ values are possible. Using the SPIRE/FTS data to select the sub-regions of the solution space that can reproduce also the high-J CO lines selects regions with $\mathrm{T}_{\text {kin }} \geqslant 70 \mathrm{~K}$ that still include Galactic $\mathrm{X}_{\mathrm{CO}}$ values, and point towards what may be a systematic underestimate of molecular gas mass in ULIRGs (Papadopoulos et al. 2012a).

\section{Acknowledgement}

The project was funded by the John S. Latsis Public Benefit Foundation. The sole responsibility for the content lies with its authors.

\section{References}

Aalto S., Booth R. S., Black J. M., \& Johansson L. E. B. 1995, A\& A, 300, 369

Braine J. \& Combes F. 1992, A\&GA, 264, 433

Bryant P. M. \& Scoville N. Z. 1996, ApJ, 457, 678

Dickman R. L., Snell R. L. \& Schloerb F. P. 1986, ApJ, 309, 326

Downes D. \& Solomon, P. M. 1998, ApJ, 507, 615

Gao Y. \& Solomon P. M. 2004, ApJ, 606, 271

Hollenbach D. \& Tielens A. G. G. M. 1999, Rev. of Mod. Physics, Vol. 71, pg. 173

Mao R. Q., Schulz A., Henkel C., et al. 2010, ApJ, 724, 1336

Maloney P. M., \& Black J. H. 1988, ApJ, 325, 389

Papadopoulos P. P., Thi W.-F., \& Viti S. 2002, ApJ, 579, 270

Papadopoulos P. P. 2007, ApJ, 656, 792

Papadopoulos P. P., van der Werf P., Xilouris E., Isaak K. G., \& Gao Y. 2012a, ApJ, 751, 10

Papadopoulos P. P., van der Werf P., Xilouris E., Isaak K. G., Gao Y., \& Muehle S. 2012b, MNRAS (in press, arXiv:1109.4176)

Sakamoto S., Hayashi M., Hasegawa T., Handa T. \& Oka T. 1994 ApJ, 425, 641

Sanders D. B. Mazzarella J. M., Kim D.-C., Surace J. A., \& Soifer B. T. 2003, AJ, 126, 1607

Solomon P. M. \& Barrett J. W. 1991, in: F. Combes \& F. Casoli. (eds.), Dynamics of Galaxies and Their Molecular Cloud Distributions, Proc. IAU Symposium No. 146, p. 235

Solomon P. M., Downes D., Radford S. J. E., \& Barrett J. W. 1997, ApJ, 478, 144

Soifer B. T., Sanders D. B., Madore B. F., et al. 1987, ApJ, 320, 238

Wolfire M. G., Tielens A. G. G. M. \& Hollenbach D. 1990, ApJ, 358, 116

Wolfire M. G., Hollenbach D., \& Tielens A. G. G. M. 1993, ApJ, 402, 195

Wolfire M. G., Hollenbach D., \& McKee C. F. 2010, ApJ, 716, 1191

Yao L., Seaquist E. R., Kuno N., \& Dunne L. 2003, ApJ, 588, 771

Young J. S. \& Scoville N. Z. 1991, ARA\&A 29, 581 